\title{
Labyrinthe
}

32 | 2009 (1)

Le petit théâtre intellectuel

\section{Carlo Ginzburg. Historic Strip}

\section{Pierre Savy}

\section{OpenEdition}

\section{Journals}

Édition électronique

URL : http://journals.openedition.org/labyrinthe/3997

DOI : $10.4000 /$ labyrinthe.3997

ISSN : 1950-6031

Éditeur

Hermann

Édition imprimée

Date de publication : 19 juin 2009

Pagination : 83-87

ISBN : 978-2-7056-6885-3

\section{Référence électronique}

Pierre Savy, «Carlo Ginzburg. Historic Strip », Labyrinthe [En ligne], $32 \mid 2009$ (1), mis en ligne le 01

février 2011, consulté le 19 avril 2019. URL : http://journals.openedition.org/labyrinthe/3997 ; DOI :

10.4000/labyrinthe.3997

Propriété intellectuelle 


\section{CARLO GINZBURG. HISTORIC STRIP}

Carlo Ginzburg est une figure complexe: non pas, cela va sans dire, celle d'un savant hyperspécialisé et isolé; pas seulement celle d'un historien brillant et érudit, d'un élégant Conférencier; et pas non plus celle d'un " penseur engagé », d'un intellectuel universel à l'ancienne. On a, à propos de son œuvre, organisé des colloques, écrit des livres, tourné des films; on raconte et il raconte qu'il travaille sur ceci ou sur cela (Dante, Jean-Pierre Purry, Bataille, etc.). Essayons de voir, à partir de ses travaux et des entretiens que, privilège de la gloire ou signe du temps, il accorde en assez grand nombre, quelle est la posture intellectuelle qu'il adopte; essayons, quoiqu'on le sache méfiant face à ce qu'ont de téléologique pareilles reconstructions, de décrire sa « trajectoire».

Il affirme qu'il voulut d'abord être romancier, puis peintre. Étudiant les humanités à Pise, il hésitait sur la discipline qu'il choisirait, et finit par devenir historien. Mais « devint »-il vraiment historien? L’idée de mouvement le définit mieux peut-être que celle d'une appartenance statique. Il demeure pourtant avant tout un historien, que son style particulier - le goût des rapprochements inattendus et des ellipses, un texte éclaté en courts chapitres, voire en paragraphes disjoints -, qu'il dit emprunté à Luigi Einaudi et qu'il rapproche du montage cinématographique, rend identifiable; un historien, dont les qualités d'intelligence, dont l'esprit pénétrant, dont la liberté alliée à l'érudition - mélange des sujets et des lieux étudiés, mais aussi des temps et même des disciplines - doivent, croyons-nous, frapper tout lecteur. Bien sûr, il continue d'incarner aux yeux du public la micro-histoire, ce projet collectif apparu sans école ni orthodoxie en Italie dans les années 1970; il la pratique même encore à l'occasion, mais fut le défenseur d'une conception « faible » de celle-ci, c'est-à-dire compatible avec l'approche macro. Il s'est intéressé aux sorcières, à partir des procès de l'Inquisition. Son œuvre ne se limite pas à sa publication la plus célèbre, Le fromage et les vers, sur la cosmologie du meunier frioulan Menocchio. Il y avait eu, dix ans avant, son premier livre, Les batailles nocturnes, sur les « benandanti», porteur d'une 
comparaison fameuse, pas tout à fait aboutie et très débattue, entre ces contre-sorciers et les chamans. Puis des travaux rencontrant l'histoire de l'art, d'autres publications d'histoire culturelle et « religieuse »-Le sabbat des sorcières notamment, qui, comme les « benandanti» et Menocchio, suscita débats et désaccords. Dès le début était à l'œuvre sa méthode, présentée dans un article célèbre sur le « paradigme indiciaire » comme proche de celles du détective ou du chasseur - affût, flair, coup de chance. Était présent aussi ce qui fut par la suite une constante: la sympathie pour les petits, pour ceux que l'on a fait taire, la volonté, sinon d'être leur porte-parole, du moins de retrouver et de faire entendre leur voix, de répondre à la question brechtienne par laquelle commence la préface du Fromage: «Qui a construit la Thèbes aux sept portes? » Ginzburg a toujours travaillé sur les phénomènes "périphériques », reléguant $a$ contrario aux marges, par le même mouvement, la " grande histoire », l'histoire-bataille. Mais son œuvre n'est sans doute pas si dissensuelle que cela : cette position d'outsider s'intéressant aux outsiders, mais récupéré - la rançon du succès -, ne conteste guère l'ordre politique dans ses fondements. Parfaitement fidèle à l'esprit des années 1970, l'attention qu'il porte aux « oubliés de l'histoire » s'est aussi révélée fort compatible avec le paysage historiographique américain - ce qui explique sans doute, en tout ou partie, que la carrière de celui qui pouvait sembler marginal en Europe se soit largement accomplie, et avec succès, aux États-Unis : après les années italiennes (surtout à Rome et Bologne), il fut longtemps professeur en Californie, et c'est depuis peu qu'il est professeur à Pise.

Le rapport de Ginzburg avec la discipline historique a pu surprendre : cet historien excède la figure de l'historien et peut sembler, influencé qu'il est par l'anthropologie, presque anti-historien; d'où sa position un peu marginale dans le champ professionnel - et d'où sa carrière, tout sauf rectiligne. Deux exemples : premièrement, son regard sur la polémique ancienne entre historiens et sceptiques a pu changer, et, sans se résigner à tout remettre à l'intuition et à l'impressionnisme, il défend une position complexe - et qui a évolué - sur le rapport au narrativisme et au postmodernisme (voir en dernier lieu Un seul témoin). Deuxièmement, lui qui a manifesté un intérêt soutenu pour la question du statut de la preuve, il admet que certaines de ses hypothèses sont difficiles à prouver, mais tient à son concept de " morphologie » (Mythes, emblèmes, traces), soit l'adoption d'une perspective atemporelle et achronologique, qui permet 
des rapprochements "morphologiques » (disons en gros structuraux) parfois osés. Depuis une vingtaine d'années, autant que d'histoire, c'est d'épistémologie qu'il s'est occupé, en publiant livres et articles importants sur les principaux thèmes qu'il a traversés : la preuve, le récit, l'observation sont au cœur d'À distance et de Rapports de force, les notions de vérité et de fiction au cœur de son dernier recueil d'articles (Il filo e le tracce). Et puis l'on sait que Ginzburg, en perpétuel mouvement, s'intéresse de plus en plus à la littérature : le terme d'une trajectoire circulaire le ramenant à ses premières amours? Les rapports entre littérature (fiction) et histoire semblent désormais son premier objet d'étude - et, dans Nulle île n'est une île, il est même question, pour ainsi dire, de littérature (anglaise) tout court: nombreux sont les savants qui, leur carrière bien entamée et bien engagée, se consacrent aux lettres, comme critique voire comme romancier (ce n'est pas le cas de Ginzburg), comme si la renommée et la liberté qu'elle offre leur permettaient de s'élever à d'autres considérations.

L'homme s'est peu à peu imposé, jusqu'à devenir, il y a une dizaine d'années - peut-être est-il un peu moins visible, depuis quelque temps - , une des figures les plus respectées et aussi les plus polyvalentes du monde universitaire à la fois européen et américain : cette position découle d'abord du fait qu'il est reconnu comme un historien novateur. Mais c'est aussi, désormais, en qualité d' " intellectuel engagé » à sa manière qu'il est connu, prenant position là où on ne l'attend pas, s'évertuant à observer le monde d'une façon différente, voire nouvelle. Son tour de force est peut-être, autant que dans l'édification d'une œuvre, dans cette évolution de sa figure: le micro-historien italien est devenu un « auteur occidental ». Ginzburg s'est éloigné progressivement des positions professionnelles qu'il occupait à ses débuts; il a emprunté le chemin d'un graduel « allégement », il a laissé s'imposer une subjectivité. De l'histoire (micro) culturelle mais « lourde », de l'étude des procès, il dériva vers l'histoire de l'art (Piero della Francesca, plus récemment Picasso, etc.), vers l'épistémologie, ensuite la politique et l'intervention, enfin semblet-il la littérature, comme on l'a dit. Il n'est pas et refuse d'être un sage universel: engagé dans les débats de l'heure, il demeure un érudit et prend toujours du recul - il se met $\grave{A}$ distance, comme l'indique le titre d'un de ses livres; il s'efforce de se tenir sur la corde. Malgré sa renommée, il ne verse pas dans la «toutologie » (il vise le tuttologo italien, celui qui parle sur tout); mal à l'aise avec le mot d' « engagement », il persiste à 
ne pas se laisser trop engager dans les media, à résister; il espère être plutôt « compétent » que « Spécialiste ».

Bref, un « intellectuel », mais qui accepte et assume le caractère non contemporain de l'histoire - on dit que, quand il enseignait à Rome, dans les années 1960, face à des étudiants désireux de présent, d'histoire utilisable, d'action politique, il défendait l'érudition historique. Ses " interventions » sont en nombre limité, et toujours exigeantes - il n'y a pas chez Ginzburg, qui ambitionne de s'adresser à la fois aux "spécialistes » et à un plus large public sans sacrifier la rigueur scientifique, de distinguo net entre les textes du savant et les textes d'intervention (comme chez d'autres: Pierre Bourdieu, Alain Badiou, etc.). Toujours l'historien demeure, qui rappelle la complexité et le caractère contradictoire du passé, qui tisse érudition historique et considérations politiques. Il parle surtout du passé, mais d'un air de dire que ce passé nous parle du présent, et il parle aussi directement du présent: ça n'est pas d'hier, du reste - voir sa dénonciation des sympathies de Georges Dumézil pour la culture nazie. Il a aussi écrit sur «Un lapsus du pape Wojtila » (l'article est repris dans $A$ distance), ou sur l'affaire Adriano Sofri - Le juge et l'historien dénonçait le procès en terrorisme fait sur des bases fragiles à cet homme d'extrêmegauche. Ce procès lui fit une sensation familière, tant il ressemblait à un procès d'Inquisition, ce qui le convainquit d'écrire pour son ami, qu'il croit innocent, ce livre d'engagement qui est aussi une réflexion sur les rapports entre la figure du juge et celle de l'historien et leur position face à la preuve - en somme, une œuvre bien représentative de la façon qu'a Ginzburg de concevoir l'intervention. Mais l'échec pratique de son livre (Sofri resta en prison), qui correspondait sans doute au paroxysme de son intervention, a confirmé à ses yeux l'opportunité d'une position médiane, qu'il occupe avec talent.

P. S.

\section{Bibliographie, références, lectures}

I. Ouvrages de Carlo Ginzburg (nous ne signalons que les livres que nous avons évoqués): Les Batailles nocturnes, Lagrasse, Verdier, 1980; Le Fromage et les vers, Paris, Flammarion, 1980; Mythes, emblèmes, traces, Paris, Flammarion, 1989; Le Sabbat des sorcières, Paris, Gallimard, 1992; 


\section{Carlo Ginzburg. Historic Strip}

Le Juge et l'historien, Lagrasse, Verdier, 1997; Un seul témoin, Paris, Bayard, 2007; À distance, Paris, Gallimard, 2001; Rapports de force, Paris, Gallimard/Le Seuil, 2003; Nulle île n'est une île, Lagrasse, Verdier, 2005; Il filo e le tracce, Milan, Feltrinelli, 2006.

II. Textes avec Carlo Ginzburg: " On the dark side of history » (www.eurozine.com/articles/2003-07-11-ginzburg-en.html, consulté le 14 février 2009); « De près, de loin. Des rapports de force en histoire », Vacarme, 18, 2002 (www.vacarme.org/article235.html, consulté le 14 février 2009); «L'historien et l'avocat du diable », Genèses, 53, 2003, p. 113-138, et 54, 2004, p. 112-129 (www.cairn.info/load_pdf. php? ID_ARTICLE = GEN_053_0113, et www.cairn.info/load_pdf. php? ID_ARTICLE = GEN_054_0112, consultés le 14 février 2009).

III. Ouvrages sur Carlo Ginzburg: Aldo Colonnello et Andrea Del Col dir., Uno storico, un mugnaio, un libro, Trieste, 2002; Denis Thouard dir., L'interprétation des indices, Villeneuve-d'Ascq, 2007. 